\title{
Differential mRNA Expression of $C O X-2$ and Proinflammatory Mediators in Patients with Rotator Cuff Tears and Osteoarthritis of the Hip
}

\author{
Tomonori Kenmoku ${ }^{*}$, Kentaro Uchida ${ }^{1}$, Naoshige Nagura ${ }^{1}$, Hisako Fujimaki ${ }^{1}$, \\ Mitsufumi Nakawaki', Naonobu Takahira' ${ }^{2}$, Kensuke Fukushima', Ryo Tazawa1, \\ Kyoko Muneshige1, Gen Inoue1, Masashi Takaso' ${ }^{1}$
}

\footnotetext{
${ }^{1}$ Department of Orthopedic Surgery, Kitasato University School of Medicine, Sagamihara, Japan ${ }^{2}$ Department of Allied Health Science, Kitasato University School of Medicine, Sagamihara, Japan Email: *pseudolefty811@yahoo.co.jp
}

How to cite this paper: Kenmoku, T., Uchida, K., Nagura, N., Fujimaki, H., Nakawaki, M., Takahira, N., Fukushima, K., Tazawa, R., Muneshige, K., Inoue, G. and Takaso, M. (2019) Differential mRNA Expression of $C O X-2$ and Proinflammatory Mediators in Patients with Rotator Cuff Tears and Osteoarthritis of the Hip. Open Journal of Orthopedics, 9, 254-263. https://doi.org/10.4236/ojo.2019.911026

Received: October 10, 2019

Accepted: November 26, 2019

Published: November 29, 2019

Copyright () 2019 by author(s) and Scientific Research Publishing Inc. This work is licensed under the Creative Commons Attribution International License (CC BY 4.0).

http://creativecommons.org/licenses/by/4.0/ (c) (i) Open Access

\begin{abstract}
Purpose: Bursal inflammation is thought to be a major cause of pain in degenerative rotator cuff tears (RCTs). While the expression of proinflammatory mediators, such as $C O X-2$, TNF- $\alpha$, IL- $1 \beta$, and IL-6, is crucial for the pathophysiology of osteoarthritis (OA), their role in degenerative RCTs remains unknown. The aim of this study was to determine the expression of $C O X-2$ and proinflammatory mediators in the development of RCT-induced pain by comparing their levels in patients with hip OA or RCTs. Methods: We included samples obtained from 31 shoulders of 31 patients with RCTs and samples from 30 hips of 27 patients with hip OA. The mRNA levels of $C O X-2$, $T N F-\alpha, I L-1 \beta$, and $I L-6$ were determined using RT-PCR, and were compared between the subacromial bursa and hip joints. We also analyzed IL- $1 \beta$-induced $C O X-2$ expression in the subacromial bursa and synovial blast of the hip. Results: $C O X-2, I L-1 \beta$, and $I L-6$ expression levels were significantly lower in the subacromial bursa of RCTs than in hip OA samples, while no significant difference was observed for $T N F-\alpha$. No significant difference in the fold increase was observed between subacromial bursa and hip OA samples, even though IL- $1 \beta$-induced $C O X-2$ expression increased in both samples. Conclusion: Our findings suggest that the main mechanism underlying pain development differs between patients with RCTs and those with hip OA.
\end{abstract}

\section{Keywords}

Rotator Cuff Tear, Pain, Hip Osteoarthritis, Tumor Necrosis Factor- $\alpha$, Interleukin (IL)- $1 \beta, C O X-2$, IL-6 


\section{Introduction}

Most patients with rotator cuff tears (RCTs) first come to the hospital because of pain [1]. Inflammation is normally implicated as the source of shoulder pain in patients with rotator cuff impairments [2] [3]. Inflammatory processes occur in the acute phase of RCTs following tendon injury, and bursal inflammation is thought to be a major cause of shoulder pain in degenerative RCTs [2] [3] [4], particularly when compared with inflammation in the glenohumeral (GH) joint [5]. However, the expression of proinflammatory mediators in degenerative RCTs remains largely unknown.

In contrast to RCTs, the origin of pain inosteoarthritis (OA) has been extensively studied. Inflammatory stimuli initiate a cascade of events, including the release of cytokines and other mediators from the cartilage and synovium. These induce metabolic disturbances and enhance catabolism in joint tissues of patients with OA, which promotes joint pain [6]. Proinflammatory mediators, especially tumor necrosis factor-alpha (TNF- $\alpha$ ), interleukin (IL)- $1 \beta$, and IL-6, are crucial for the pathophysiology of OA [6]. In addition, anti-inflammatory effects are related to the downregulation of cyclooxygenase-2 (COX-2) and reduced proinflammatory mediator expression, regardless of joint [7] [8].

The purpose of this study was to investigate the role of $C O X-2$ and proinflammatory mediators in the development of pain in patients with degenerative RCTs. To this end, we used real-time polymerase chain reaction (RT-PCR) to compare these mediators' mRNA expression in RCT patients and patients with hip OA-associated pain. We show that $C O X-2$ and proinflammatory mediator levels were generally lower in patients with RCTs than in those with hip OAassociated pain.

\section{Methods}

\subsection{Patient Selection}

The experimental protocol was conducted in accordance with the guidelines of Kitasato University Medical Ethics Organization for Clinical Research (KMEO B13-113). The Institutional Review Board of our institute approved the protocol for this study, and all participants provided written informed consent. Between September 2013 and June 2016, we obtained samples from 31 shoulders of 31 patients undergoing arthroscopic rotator cuff repair for degenerative RCTs, and 30 hips from 27 patients undergoing total hip arthroplasty or arthroscopic hip surgery for hip OA. Inclusion criteria were: onset of joint pain more than one month before surgery and age over 20 years. Patients with traumatic RCTs, shoulder or hip fractures at the time of surgery, a history of previous shoulder or hip operations, a history of rheumatoid arthritis or other collagen diseases, and a history of corticosteroid use, including both injections and oral usage within one month before operation, were excluded from the study.

For all patients, age, sex, and clinical history were recorded. To define the severity of anatomical damage, the size of the RCT was determined by coronal 
imaging using Cofield and Deorio classification [9], whereas hip OA grade was assessed using the anteroposterior view of plain radiographs and the Kellgren and Lawrence X-ray grading system [10]. Preoperative clinical assessments of RCTs and hip OA were performed using the Constant score and modified Harris Hip Score, respectively [11] [12]. Cartilage condition was assessed arthroscopically or macroscopically according to the International Cartilage Repair Society (ICRS) classification system [13].

All arthroscopic rotator cuff repair surgeries were performed by a single experienced shoulder surgeon (K.T.). We harvested a synovial membrane sample from the subacromial space of each RCT patient because subacromial bursitis is generally accepted as the main source of pain in degenerative RCTs compared with inflammation in the GH joint [2] [3] [4] [5]. For total hip arthroplasty, every patient was operated using the anterolateral approach in the supine position by two experienced hip surgeons (T.N. and F.K.). We harvested a sample of the synovial membrane from the site of strongest synovitis for further evaluation when capsulectomy was performed.

\subsection{Isolation of RNA and Quantitative RT-PCR}

Total RNA was extracted from the harvested synovial samples using TRIzol (Invitrogen, Carlsbad, CA, USA) according to the manufacturer's instructions, and was used as template for first-strand cDNA synthesis using SuperScript III RT (Invitrogen). The PCR reaction mixture consisted of $2 \mu \mathrm{L}$ of cDNA, specific primer set $(0.2 \mu \mathrm{M}$ final concentration), and $12.5 \mu \mathrm{L}$ SYBR Premix Ex Taq (TaKaRa, Kyoto, Japan) in a final volume of $25 \mu \mathrm{L}$. The sequences of PCR primer pairs are listed in Table 1. Quantitative PCR was performed using the CFX-96 RT-PCR detection system (Bio-Rad, Hercules, CA, USA). PCR cycle parameters were as follows: initial denaturation at $95^{\circ} \mathrm{C}$ for $1 \mathrm{~min}$, followed by 40 cycles of $95^{\circ} \mathrm{C}$ for $5 \mathrm{~s}$, and $60^{\circ} \mathrm{C}$ for $30 \mathrm{~s}$. mRNA expression of target genes was normalized to that of glyceraldehyde 3-phosphate dehydrogenase (GAPDH) mRNA using delta-delta method.

Table 1. Sequences of PCR primer pairs.

\begin{tabular}{ccc}
\hline Primer & Sequence $\left(5^{\prime}-3^{\prime}\right)$ & Product size $(\mathrm{bp})$ \\
\hline COX-2-F & TGGCTGAGGGAACACAACAG & 74 \\
COX-2-R & AACAACTGCTCATCACCCCA & \\
TNF- $\alpha-\mathrm{F}$ & CTTCTGCCTGCTGCACTTTG & 118 \\
TNF- $\alpha-\mathrm{R}$ & GTCACTCGGGGTTCGAGAAG & \\
IL-6-F & GAGGAGACTTGCCTGGTGAAA & 199 \\
IL-6-R & TGGCATTTGTGGTTGGGTCA & \\
IL- $1 \beta-\mathrm{F}$ & GTACCTGTCCTGCGTGTTGA & 153 \\
IL-1 $\beta-\mathrm{R}$ & GGGAACTGGGCAGACTCAAA & \\
GAPDH-F & TGTTGCCATCAATGACCCCTT & 202 \\
GAPDH-R & CTCCACGACGTACTCAGCG &
\end{tabular}


The levels of four molecular biomarkers of inflammation in synovial tissue samples were measured: $C O X-2, T N F-\alpha, I L-6$, and $I L-1 \beta$. We compared these levels between the RCT and OA patient groups, and between the subacromial bursa (SAB) of RCTs and hip joints of hip OA.

\subsection{Effect of IL-1 $\beta$ on $C O X-2$ Expression in the Synovial Fibroblasts of SAB and Hip Synovium}

Synovial tissue was excised from the SAB and hip joints for the assessment of molecular biomarker levels, as previously described [2] [3] [14]. However, SAB is pathologically different from joint synovial blast [15], and no reports on the difference in $C O X-2$ expression between $\mathrm{SAB}$ and joint synovial blast are available. Therefore, we analyzed IL- $1 \beta$-induced $C O X-2$ expression in the SAB and synovial blast of the hip. The excised tissue was minced, digested for $2 \mathrm{~h}$ at $37^{\circ} \mathrm{C}$ with type 1 collagenase (0.1\%; Sigma-Aldrich, Lakewood, NJ, USA), and passed through a $40-\mu \mathrm{m}$ filter (Becton Dickinson, Franklin Lakes, NJ, USA), to yield single-cell suspensions. Cell numbers were determined using a hemocytometer. Nucleated cells isolated from the synovium were seeded at $1 \times 10^{4} \mathrm{cells} / \mathrm{cm}^{2}$ in 6-well plates containing minimum essential medium (Gibco, Carlsbad, CA, USA) supplemented with $10 \%$ fetal bovine serum, $100 \mathrm{U} / \mathrm{mL}$ penicillin, and 100 $\mu \mathrm{g} / \mathrm{mL}$ streptomycin, and the cells were incubated at $37^{\circ} \mathrm{C}$ in an incubator with $5 \% \mathrm{CO}_{2}$ for seven days. After one week, synovial fibroblasts were left untreated or treated with $50 \mathrm{ng} / \mathrm{mL}$ of human recombinant IL- $1 \beta$ (Biolegend, San Diego, CA, USA) for $24 \mathrm{~h}$. Cells were harvested, total RNA was isolated as described, and $C O X-2$ mRNA in synovial fibroblasts was determined by RT-PCR. The experiment was performed four times.

\subsection{Statistical Analysis}

Statistical analyses and power analyses were performed using commercial software (JMP Pro version 14.3, SAS Institute Inc., Cary NC, USA). Results are expressed as the mean \pm standard deviation (SD). The chi-square test was used for gender comparisons in RCT and hip OA groups. Mann-Whitney's U-test was used to compare age, and mRNA levels of $C O X-2$ and proinflammatory mediators between two groups. For all statistical analyses, significance was defined as $P<0.05$.

\section{Results}

\subsection{Patient Characteristics}

Patients' characteristics are presented in Table 2. There was no significant difference in age between the groups, whereas significant differences in gender and ICRS classification were observed.

\subsection{Proinflammatory Cytokine Levels in Patients with RCTs and Hip OA}

Mann-Whitney's U-test revealed a significant difference in $C O X-2, I L-6$, and 
Table 2. Patient characteristics.

\begin{tabular}{cccc}
\hline & $\begin{array}{c}\text { RCT group } \\
\text { (31 shoulder samples, } \\
\text { 31 patients) }\end{array}$ & $\begin{array}{c}\text { Hip OA group } \\
(30 \text { hip samples, } \\
27 \text { patients) }\end{array}$ & Pvalue \\
\hline Age (years) & 66 (range, $44-79)$ & 63 (range, $40-86)$ & 0.22 \\
Sex & F, 14; M, 17 & F, 23; M, 4 & 0.002 \\
Side & R, 18; L, 13 & R, 18; L, 12 & 0.88 \\
Anatomical damage & $7: 9: 5: 6: 4$ & $0: 0: 5: 25$ & - \\
Clinical score $^{*}$ & 51.9 (range, $16-85)$ & 40.5 (range, $19-65)$ & - \\
ICRS classification $^{\dagger}$ & $18: 6: 6: 1: 0$ & $0: 0: 0: 30$ & $<0.0001$ \\
\hline
\end{tabular}

RCTs, rotator cuff tears; OA, osteoarthritis; F, female; M, male; R, right; L, left; NSAID, nonsteroidal anti-inflammatory drug. ${ }^{*}$ Severity of RCTs was assessed by Cofield and Deorioclassification [9] (partial tear: small tear: moderate tear: large tear: massive tear). Severity of OA hip joint damage was assessed using plain radiographs [10] (grades 1:2:3:4). "Shoulder damage was assessed by the Constant score [11], whereaship damage was assessed using a modified Harris Hip Score [12]. ${ }^{\dagger}$ (ICRS grades 0:1:2:3:4) [13].

$I L-1 \beta$ mRNA levels between RCT and hip OA patients. However, no significant difference in $T N F-\alpha$ expression was observed between three synovial fibroblast samples (Figure 1, Table 3).

\subsection{Effect of IL- $1 \beta$ on $C O X-2$ Expression in the Synovial Fibroblasts of SAB and Hip Joint}

COX-2 mRNA expression increased in SAB samples of RCT patients and hip OA samples following treatment with IL- $1 \beta$. However, no significant difference in fold increase was observed between SAB samples from RCT and hip OA patients (fold change: $\mathrm{SAB}, 17.7 \pm 5.2$; hip $\mathrm{OA}, 19.5 \pm 12.0 ; P=0.76$; power, 0.06 ).

\section{Discussion}

The present study revealed that $C O X-2, I L-6$, and $I L-1 \beta$ mRNA levels were significantly lower in patients with RCTs than in those with hip OA, whereas no significant difference in TNF- $\alpha$ expression was detected. In addition, both SAB and hip joint synovium showed an increase, albeit non significant, in $C O X-2$ mRNA expression following treatment with IL- $1 \beta$. Hence, our findings suggest that the main mechanism underlying the development of pain in patients with RCTs after the acute phase differs from that underlying the development of pain in patients with hip OA because, with the exception of $T N F-\alpha$, the levels of critical pain mediators involved in the pathophysiology of OA were lower in RCT patients.

Even though they are both ball joints, the shoulder joint differs from the hip joint in that it is a non-weight bearing joint. The destructive response of the synovium, which is induced by cartilage breakdown, plays a major role in the development of OA pain [6]. In contrast to OA, SAB is affected by a torn rotator cuff. Our RCT subjects exhibited a much lower grade of ICRS classification compared with the hip OA group, suggesting that in their case, subacromial bursitis was not affected by cartilage debris as much as it was inhip OA. Accordingly, 


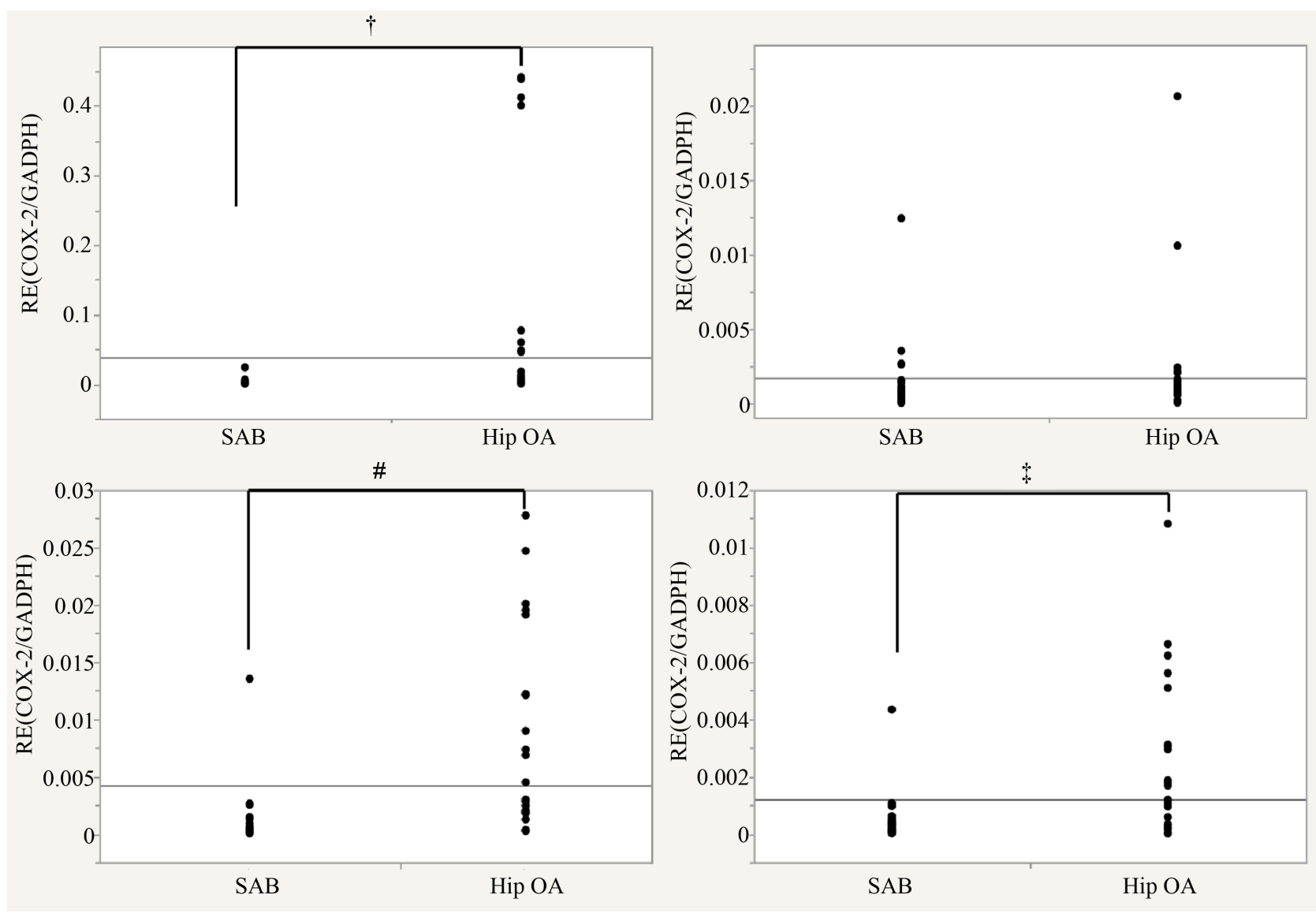

Figure 1. Proinflammatory cytokine levels in patients with RCTs and hip OA. Bars represent significant differences between subacromial bursa $(\mathrm{SAB})$ samples and hip joint samples. ${ }^{\dagger}:$ COX-2 $(P<0.0001)$; ${ }^{*}: I L-6(P<0.0001)$; $^{\ddagger}: I L-1 \beta(P=0.021)$. RE: relative expression.

Table 3. mRNA levels of proinflammatory cytokines and cyclooxygenase-2 (COX-2).

\begin{tabular}{cccc}
\hline & RCTs & Hip OA & $P$ value (power) \\
\hline$C O X-2$ & $0.0024 \pm 0.0044$ & $0.078 \pm 0.15$ & $<0.001(0.76)$ \\
$T N F-\alpha$ & $0.0014 \pm 0.0021$ & $0.0021 \pm 0.0042$ & $0.59(0.13)$ \\
$I L-6$ & $0.0001 \pm 0.0024$ & $0.0081 \pm 0.0085$ & $<0.001(0.99)$ \\
$I L-1 \beta$ & $0.0004 \pm 0.0008$ & $0.0020 \pm 0.0027$ & $0.021(0.82)$ \\
\hline
\end{tabular}

TNF- $\alpha$, tumor necrosis factor-alpha; IL-6, interleukin-6; IL-1 $\beta$, interleukin- $1 \beta$.

the significant difference in mRNA expression of $C O X-2, I L-1 \beta$, and $I L-6$ may be associated with the difference of stimulation for each synovial tissue.

TNF- $\alpha$ is considered the main pain effector in both RCTs and OA [4] [6]. TNF- $\alpha$ correlates strongly with IL- 1 or IL- 6 in articular or subacromial bursae [3] [4] [6] [16], however, TNF- $\alpha$ can also act independently to initiate or propagate inflammation [6]. In a previous animal study, we confirmed continued $T N F-\alpha$ expression over a period of two weeks, even though $C O X-2$ and $I L-1 \beta$ were significantly decreased [17]. That result may explain why only TNF- $\alpha$ mRNA levels failed to exhibit any significant difference between RCT and hip OA 
groups.

IL- $1 \beta$ is thought to be associated with pain of both RCTs and OA [2] [4] [6] [18]. The significant increasing of $I L-6$ expression has been confirmed, however, the relationship between IL-6 and RCTs pain is still controversial [2] [4] [18]. We examined IL- $1 \beta$-induced expression of $C O X-2$ in synovial fibroblasts, a treatment that induces considerable $C O X-2$ expression [7] [16] [19] [20]. However, we did not observe any significant differences in $C O X-2$ expression between $\mathrm{SAB}$ and hip synovial fibroblasts, suggesting that these cells expressed similar levels of both $C O X-2$ and its downstream effector, prostaglandin E2 (PGE2). $C O X-2$ and $I L-1 \beta$ mRNA levels decreases with time in rat RCT model [17]. Accordingly, the observed differences in $C O X-2, I L-1 \beta$, and $I L-6 \mathrm{mRNA}$ levels may not reflect differential basal levels of expression between tissues. Instead, they may indicate the difference in inflammation states observed in these diseases.

In spite of their frequent prescription in this context, the effectiveness of nonsteroidal anti-inflammatory drugs (NSAIDs) in the management of pain in patients with degenerative RCTs is controversial [21] and not recommended for the conservative treatment and perioperative management of RCTs [22]. In contrast, NSAIDs are strongly recommended for the management of hip OA pain [23] [24], as $C O X-2$ and other inflammation mediators are produced in the synovial tissues of OA joints [14] [25]. The binding of NSAIDs to COX isozymes inhibits the synthesis of prostanoids [26] [27] [28]. PGE2 is the dominant prostanoid produced during inflammation, and inhibition of its synthesis by NSAIDs is believed to be the main mechanism underlying potent analgesic and anti-inflammatory properties of these agents [26] [27] [28]. Moreover, the efficacy with which pain and PGE2 production are inhibited depends on the dose of NSAIDs [25]. Hence, if pain correlates with $C O X-2$ expression, a normal dose of oral NSAIDs can be sufficient to control both OA and RCT pain. Our findings suggest that degenerative RCT-associated pain depends on factors other than $C O X-2$. Thus patients with RCTs, who do not feel any efficacy of taking NSAIDs, may not benefit from their use for pain management. Moreover, discontinuing NSAID treatment would allow such patients to avoid the unwanted side effects caused by these drugs. However, in cases where NSAIDs are efficacious to the patient with RCTs, their use for pain management can be kept in place.

\section{Limitations}

The major limitation of this study was the impossibility to compare mRNA expression of proinflammatory mediators between RCTs and shoulder OA, as rotator cuff degeneration occurs spontaneously in patients with shoulder OA. The target of pain control medication is to lower the level of pain-related substance rather than that of pain inducers. Our purpose was to compare the mRNA expression patterns of proinflammatory cytokines in synovial tissues between RCTs and hip OA. To this end, our study clearly shows the difference in mRNA 
expression of proinflammatory mediators induced by the different forms of stimulation.

Another limitation was our failure touse enzyme-linked immunosorbent assay (ELISA) or western blotting to support RT-PCR data. Assessment of COX-2 expression by RT-PCR is considered appropriate to determine the activity of this enzyme in synovial tissues, as it is consistent with immunohistochemical data [15]. In addition, the amount of PGE2 measured by ELISA is related to the expression of $C O X-2, T N F-\alpha$, and $I L-1 \beta$ measured by RT-PCR [7] [8]. Therefore, $C O X-2, T N F-\alpha, I L-6$, and $I L-1 \beta$ levels assessed using RT-PCR are consistent with the detection of these four molecular biomarkers of inflammationby ELISA or western blotting.

A minor limitation is constituted by the significant difference in gender between RCT and hip OA groups due to higher prevalence of hip dysplasia among females [29]. However, we could not detect any difference in mRNA expression levels of $C O X-2, T N F-\alpha, I L-6$, and $I L-1 \beta$ nor in the efficacy of NSAIDs or corticosteroids between genders. Therefore, we believe that the different gender composition between RCT and hip OA groups had no bearing on our results.

\section{Conclusion}

We compared the mRNA expression of $C O X-2$ and proinflammatory mediators in patients with degenerative RCTs and hip OA, and demonstrated that $C O X-2$, $I L-1 \beta$, and $I L-6$ levels in SAB samples obtained from patients with RCTs were significantly lower than those from patients with hip OA. Our findings suggest that the main mechanism underlying the development of pain in patients with degenerative RCTs differs from that underlying the development of pain in patients with hip OA.

\section{Funding}

This study was supported by research grants from Japan Society for the Promotion of Science (JSPS) Grant-in-Aid for Scientific Research (KAKENHI) Grant No. 18 K 16633.

\section{Conflicts of Interest}

The author(s), their immediate families, and any research foundations with which they are affiliated have not received any financial payment or other benefits from any commercial entity related to the subject of this article.

\section{References}

[1] Itoi, E., Minagawa, H., Yamamoto, N., et al. (2006) Are Pain Location and Physical Examinations Useful in Locating a Tear Site of the Rotator Cuff? The American Journal of Sports Medicine, 34, 256-264. https://doi.org/10.1177/0363546505280430

[2] Gotoh, M., Hamada, K., Yamakawa, H., et al. (2001) Interleukin-1-Induced Subacromial Synovitis and Shoulder Pain in Rotator Cuff Diseases. Rheumatology (OX- 
ford), 40, 995-1001. https://doi.org/10.1093/rheumatology/40.9.995

[3] Voloshin, I., Gelinas, J., Maloney, M.D., et al. (2005) Proinflammatory Cytokines and Metalloproteases Are Expressed in the Subacromial Bursa in Patients with Rotator Cuff Disease. Arthroscopy, 21, 1076.e1-1076.e5.

https://doi.org/10.1016/j.arthro.2005.05.017

[4] Blaine, T.A., Kim, Y.S., Voloshin, I., et al. (2005) The Molecular Pathophysiology of Subacromial Bursitis in Rotator Cuff Disease. Journal of Shoulder and Elbow Surgery, 14, 84S-89S. https://doi.org/10.1016/j.jse.2004.09.022

[5] Gotoh, M., Hamada, K., Yamakawa, H., et al. (2002) Interleukin-1-Induced Glenohumeral Synovitis and Shoulder Pain in Rotator Cuff Diseases. Journal of Orthopaedic Research, 20, 1365-1371. https://doi.org/10.1016/S0736-0266(02)00063-3

[6] Kapoor, M., Martel-Pelletier, J., Lajeunesse, D., et al. (2010) Role of Proinflammatory Cytokines in the Pathophysiology of Osteoarthritis. Nature Reviews Rheumatology, 7, 33-42. https://doi.org/10.1038/nrrheum.2010.196

[7] Choi, Y.J., Lee, W.S., Lee, E.G., et al. (2014) Sulforaphane Inhibits IL-1 $\beta$-Induced Proliferation of Rheumatoid Arthritis Synovial Fibroblasts and the Production of MMPs, COX-2, and PGE2. Inflammation, 37, 1496-1503.

https://doi.org/10.1007/s10753-014-9875-4

[8] Maghsoudi, H., Hallajzadeh, J. and Rezaeipour, M. (2018) Evaluation of the Effect of Polyphenol of Escin Compared with Ibuprofen and Dexamethasone in Synoviocyte Model for Osteoarthritis: An in Vitro Study. Clinical Rheumatology, 37, 24712478. https://doi.org/10.1007/s10067-018-4097-z

[9] De Orio, J. and Cofield, R. (1984) Results of a Second Attempt at Surgical Repair of a Failed Initial Rotator-Cuff Repair. The Journal of Bone and Joint Surgery. American Volume, 66, 563-567. https://doi.org/10.2106/00004623-198466040-00011

[10] Kellgren, J. and Lawrence, J. (1957) Radiological Assessment of Osteoarthritis. Annals of the Rheumatic Diseases, 16, 494-502. https://doi.org/10.1136/ard.16.4.494

[11] Constant, C.R. and Murley, A.H. (1987) A Clinical Method of Functional Assessment of the Shoulder. Clinical Orthopaedics and Related Research, 214, 160-164. https://doi.org/10.1097/00003086-198701000-00023

[12] Harris, W.H. (1969) Traumatic Arthritis of the Hip after Dislocation and Acetabular Fractures: Treatment by Mold Arthroplasty. The Journal of Bone and Joint Surgery. American Volume, 51, 737-755. https://doi.org/10.2106/00004623-196951040-00012

[13] Mainil-Varlet, P., Aigner, T. and Brittberg, M. (2003) Histological Assessment of Cartilage Repair. A Report by the Histology Endpoint Committee of the International Cartilage Repair Society. The Journal of Bone and Joint Surgery. American Volume, 85, 45-57. https://doi.org/10.2106/00004623-200300002-00007

[14] Siegle, I., Klein, T., Backman, J.T., et al. (1998) Expression of Cyclooxygenase 1 and Cyclooxygenase 2 in Human Synovial Tissue. Arthritis \& Rheumatology, 41, 122-129. https://doi.org/10.1002/1529-0131(199801)41:1<122::AID-ART15>3.0.CO;2-8

[15] Smith, M.D. (2011) The Normal Synovium. The Open Rheumatology Journal, 5, 100-106. https://doi.org/10.2174/1874312901105010100

[16] Shindle, M.K., Chen, C.C.T., Robertson, C., et al. (2011) Full-Thickness Supraspinatus Tears Are Associated with More Synovial Inflammation and Tissue Degeneration than Partial-Thickness Tears. Journal of Shoulder and Elbow Surgery, 20, 917-927. https://doi.org/10.1016/j.jse.2011.02.015

[17] Nagura, N., Kenmoku, T., Uchida, K., et al. (2019) Nerve Growth Factor Conti- 
nuously Elevates in a Rat Rotator Cuff Tear Model. Journal of Shoulder and Elbow Surgery, 28, 143-148. https://doi.org/10.1016/j.jse.2018.06.030

[18] Shin, C.A., Wu, K.C., Shao, C.J., et al. (2018) Synovial Fluid Biomarkers: Association with Chronic Rotator Cuff Tea Severity and Pain. Journal of Shoulder and Elbow Surgery, 27, 545-552. https://doi.org/10.1016/j.jse.2017.09.020

[19] Nagura, N., Uchida, K., Kenmoku, T., et al. (2019) IL-1 $\beta$ Mediates NGF and COX-2 Expression through Transforming Growth Factor-Activating Kinase 1 in Subacromial Bursa Cells Derived from Rotator Cuff Tear Patients. Journal of Orthopaedic Science, 24, 925-929. https://doi.org/10.1016/j.jos.2019.02.006

[20] O’Neill, L.A.J., Barrett, M.L. and Lewis, G.P. (1987) Induction of Cyclo-Oxygenase by Interleukin-1 in Rheumatoid Synovial Cells. FEBS Letters, 212, 35-39. https://doi.org/10.1016/0014-5793(87)81551-X

[21] Inderhaug, E., Kollevold, K.H., Kalsvik, M., et al. (2015) Preoperative NSAIDs, Non-Acute Onset and Long-Standing Symptoms Predict Inferior Outcome at Long-Term Follow-Up after Rotator Cuff Repair. Knee Surgery, Sports Traumatology, Arthroscopy, 25, 2067-2072. https://doi.org/10.1007/s00167-015-3845-8

[22] Pedowitz, R.A., Yamaguchi, K., Ahmad, C.S., Burks, R.T., Flatow, E.L., Green, A., et al. (2011) Optimizing the Management of Rotator Cuff Problems. The Journal of the American Academy of Orthopaedic Surgeons, 19, 368-379. https://doi.org/10.5435/00124635-201106000-00007

[23] Hochberg, M.C., Altman, R.D., April, K.T., et al. (2012) American College of Rheumatology 2012 Recommendations for the Use of Nonpharmacologic and Pharmacologic Therapies in Osteoarthritis of the Hand, Hip, and Knee. Arthritis Care \& Research, 64, 465-474. https://doi.org/10.1002/acr.21596

[24] Zhang, W., Nuki, G., Moskowitz, R.W., et al. (2010) OARSI Recommendations for the Management of Hip and Knee Osteoarthritis. Part III: Changes in Evidence Following Systematic Cumulative Update of Research Published through January 2009. Osteoarthritis and Cartilage, 18, 476-499. https://doi.org/10.1016/j.joca.2010.01.013

[25] Crofford, L.J. (1999) COX-2 in Synovial Tissues. Osteoarthritis and Cartilage, 7, 406-408. https://doi.org/10.1053/joca.1999.0226

[26] Noguchi, M., Kimoto, A., Kobayashi, S., Yoshino, T., Miyata, K. and Sasamata, M. (2005) Effect of Celecoxib, a Cyclooxygenase-2 Inhibitor, on the Pathophysiology of Adjuvant Arthritis in Rat. European Journal of Pharmacology, 513, 229-235. https://doi.org/10.1016/j.ejphar.2005.01.058

[27] Patrono, C., Patrignani, P. and García Rodríguez, L.A. (2001) Cyclooxygenase-Selective Inhibition of Prostanoid Formation: Transducing Biochemical Selectivity into Clinical Read-Outs. Journal of Clinical Investigation, 108, 7-13. https://doi.org/10.1172/JCI200113418

[28] Vane, J.R. (1971) Inhibition of Prostaglandin Synthesis as a Mechanism of Action for Aspirin-Like Drugs. Nature: New Biology, 231, 232-235. https://doi.org/10.1038/newbio231232a0

[29] Jingushi, S., Ohfuji, S., Sofue, M., et al. (2011) Osteoarthritis Hip Joint in Japan: Involvement of Acetabular Dysplasia. Journal of Orthopaedic Science, 16, 156-164. https://doi.org/10.1007/s00776-011-0025-7 